\title{
PRESERVAÇÃo DIGITAL EM COLEÇõés BIBLIOGRAFICAS DA BIODIVERSIDADE: O CASO DA BIODIVERSITY HERITAGE LIBRARY NO MUSEU PARAENSE EMÍLIO GOELDI (MPEG)
}

\author{
DIGITAL PRESERVATION IN BIBLIOGRAPHIC COLLECTIONS OF BIODIVERSITY: \\ THE CASE OF BIODIVERSITY HERITAGE LIBRARY AT THE GOELDI MUSEUM (MPEG)
}

\section{Jetur Lima de Castro ${ }^{1}$ Alessandra Nunes de Oliveira ${ }^{2}$}

\begin{abstract}
RESUMO
Relata a experiência do "Projeto de digitalização e publicação online da coleção de Obras Raras Essenciais em Biodiversidade do Museu Paraense Emílio Goeldi" (MPEG). A iniciativa é da Biodiversity Heritage Library (BHL), cuja finalidade é subsidiar, o fortalecimento e desenvolvimento da infraestrutura de informação e comunicação científica, em biodiversidade e meio ambiente. O objetivo de estudo é mostrar o projeto de digitalização e publicação online da coleção de Obras Raras Essenciais em Biodiversidade do Museu Paraense Emílio Goeldi e estudar o papel da preservação digital no acervo bibliográfico especial, utilizando os critérios de preservação digital e as políticas de digitalização. Permitindo, a construção de um esquema conceitual para agregar e conservar a informação em formato digital, dando acesso imediato, por meio das redes e fontes de informação, nacionais e internacionais. O percurso metodológico adotado foi realizado, por meio de uma pesquisa exploratória de caráter descritivo, com base na literatura como em livros, periódicos, dissertações, teses e artigos científicos já publicados abordando a temática. Deste modo, é analisado as questões mais relevantes no estudo, com relação aos métodos relacionados com a preservação de um acervo bibliográfico em formatos digitais. Neste sentido, a digitalização é aliada à preservação digital na coleção em biodiversidade, e trará enorme contribuição à biblioteca do MPEG, tornando as informações cientificas mais acessíveis, dando continuidade à memória e certificando a absoluta excelência dos suportes digitais, abarcada pelas inovações tecnológicas que remediarão suas perdas futuras.
\end{abstract}

PALAVRAS-CHAVE: Preservação digital. Digitalização de documentos. Informação científica. Biodiversidade.

\begin{abstract}
Reports the experience of the project of digitization and online publication of Essential rare works collection in biodiversity of the Museu Paraense Emílio Goeldi (MPEG). The initiative is the Biodiversity Heritage Library (BHL), whose purpose is to subsidize, the strengthening and development of scientific information and communication infrastructure, biodiversity and the environment. The purpose of the study is to show the project of digitization and online publication of Essential rare works collection in biodiversity of the Museu Paraense Emílio Goeldi and study the role of digital preservation in the bibliographic collection special, using the criteria
\end{abstract}

\footnotetext{
${ }^{1}$ Estudante de Biblioteconomia da Universidade Federal do Pará - Campus Belém. Pesquisador/colaborador da Rede Brasileira de Serviços de Preservação Digital - IBICT/Brasília. E-mail: jetur.er@gmail.com

2 Estudante de Biblioteconomia da Universidade Federal do Pará - Campus Belém. E-mail: alessandranunesoliveira@gmail.com
}

Enviado em: 04/11/2014 - Aceito em: 08/05/2015 
of digital preservation and digitization policies. Allowing the construction of a conceptual schema to add and save the information in digital format, giving immediate access, by means of networks and sources of information, both national and international. The methodological path was conducted through an exploratory research of descriptive character, based on literature as in books, journals, dissertations, theses and scientific articles already published addressing the theme. Thus, the most relevant issues is examined in the study, with related methods with the preservation of a bibliographic collection in digital formats. In this sense, the scan is combined with digital preservation in the collection on biodiversity, and will bring enormous contribution to the library of MPEG, making scientific information more accessible, giving continuity to the memory and making sure the absolute excellence of digital supports, covered by technological innovations that remedied their future losses.

KEYWORDS: Digital preservation. Scanning documents. Scientific information. Biodiversity.

\section{INTRODUÇÃO}

Com o advento das Tecnologias de Informação e Comunicação (TIC) e a relação dos processos econômicos, sociais e políticos. A sociedade encontra-se profundamente ligada a uma gama de possibilidades entre ferramentas e mecanismos de acesso livre a informação. Deste modo, em meio a este cenário o meio social vivencia uma tendência maior em relação à produção de documentos em formato digital com uma grande diversidade de informações.

Desta forma, essas informações estão elencadas na sociedade globalizada. E são organizadas em vários suportes sendo físico e digital, tendem a se submergirem ao longo de seu tempo. A partir deste aspecto, Santos; Innareli e Sousa (2009, p. 22), afirmam que seja "fundamental a iniciativa de pesquisar temas relacionados às políticas de preservação [...] Tendo em vista um projeto que proteja a preservação destes documentos e informações para que não corramos os ricos de viver numa sociedade sem memória” [...].

Diante disto, a pesquisa mostra o processo de digitalização das Obras Raras no Museu Emílio Goeldi, na qual está relacionada ao projeto de Digitalização e Publicação Online de Coleções de Obras Raras Essenciais em Biodiversidade em Bibliotecas Brasileiras. Como objetivo, o projeto tem de tratar, recuperar, disseminar e cultivar o conhecimento referente ao estudo da biodiversidade brasileira de modo a introduzir e facilitar o ensino, a pesquisa e também a gestão de políticas públicas em biodiversidade. Do mesmo modo, promovendo a disponibilidade de acesso livre as publicações para sociedade.

O projeto "Digitalização e Publicação Online de uma Coleção de Obras Raras Essenciais em Biodiversidade das Bibliotecas Brasileiras" integra uma iniciativa nacional mais abrangente de gestão do conhecimento e na informação científica em biodiversidade e meio ambiente.

O Museu Paraense Emílio Goeldi (MPEG) é um dos sete institutos nomeados para integrar e dá início ao trabalho. Sendo selecionado o acervo bibliográfico de obras raras essenciais em biodiversidade do Museu Paraense Emílio Goeldi, para ser o campo de estudo do presente trabalho. Pois, o acervo em biodiversidade representa um importante tesouro para a memória da ciência na Amazônia. 
Como objetivo geral pretendeu-se estudar o papel da preservação digital e o processo de digitalização do acervo bibliográfico especial e mostrar o caso do projeto de digitalização e publicação online da coleção de Obras Raras Essenciais em Biodiversidade do Museu Paraense Emílio Goeldi de iniciativa da Biodiversity Heritage Library.

Quanto à natureza e de acordo com os objetivos propostos o caráter metodológico deste trabalho corresponde a uma pesquisa exploratório-descritiva. Gil (2008) afirma que a pesquisa exploratória: proporcionar maior familiaridade com o problema (explicitá-lo). Pode envolver levantamento bibliográfico, entrevistas com pessoas experientes no problema pesquisado. A pesquisa exploratória apoia-se em determinados princípios bastante difundidos: 1) a aprendizagem melhor se realiza quando parte do conhecido; 2) deve-se buscar sempre ampliar o conhecimento e 3) esperar respostas racionais pressupõe formulação de perguntas também racionais (PIOVESAN, 1968).

Inicialmente, foi realizada uma pesquisa bibliográfica ou de fontes primárias e secundárias com o objetivo de localizar conteúdos em livros, periódicos, dissertações, teses e artigos científicos já publicados abordando a temática onde se terá como Trujillo Ferrari (1974, p. 230) menciona, “[...] o reforço paralelo na análise de suas pesquisas ou manipulação de suas informações".

Diante dessa afirmação Babbie (1983), mostra que a presente pesquisa é determinada como um estudo exploratório, que apresenta características de um estudo descritivo uma vez que, foi analisado e, depois foram detalhados os elementos da preservação digital e o processo de digitalização na biblioteca do Museu Paraense Emílio Goeldi.

Considerando o fato de que a curta vida média dos documentos é uma das principais ameaças fundamental para a preservação digital. O referencial teórico é formado a partir da produção de uma breve revisão de literatura construída a partir dos objetivos elencados anteriormente, onde a bibliografia relevante sobre o assunto "[...] oferece meios para definir, resolver, não somente problemas já conhecidos, como também explorar novas áreas, onde os problemas ainda não se cristalizaram suficientemente" (MANZO, 1971, p. 32, tradução nossa). Dessa forma, a pesquisa bibliográfica busca levantar informações a importância da digitalização e preservação digital em acervos raros e especiais, bibliotecas, ressaltando o projeto BHL no Museu Paraense Emílio Goeldi.

Conclui-se de forma objetiva, a importância que a preservação de documentos, bibliografias continuam a ser deliberado pela disposição do objeto informacional, servir às utilizações que lhe são atribuídas, e às suas pertinências para garantir a continuidade do conhecimento. Deste modo, relata a experiência frente a processo de digitalização junto a propostas de preservação digital na Biblioteca Domingos Soares Ferreira Penna, coordenada por Maria Astrogilda Ribeiro da Coordenação de Informação e Documentação do MPEG. E descreve as atividades regidas das etapas da digitalização das Obras Raras em Biodiversidade, ações práticas do processo de gerenciamento da digitalização e as políticas de digitalização a partir do aparato conceitual da preservação digital. 


\section{PRESERVAÇÃO DIGITAL: UM POUCO DE CONCEITO}

Com o início da revolução tecnológica e científica, que adveio após a Segunda Guerra Mundial, o conhecimento sofreu um processo de crescimento, versado como a explosão informacional, acarretando apreciáveis mudanças no processo de registro, armazenamento, transmissão e acesso a informação (SARACEVIC, 1996). Apesar dos processos de desenvolvimento das formas de difusão da informação, nasce para nós um novo paradigma, que sobretudo traz a importância dos novos suportes em que se aloca a informação. A informação digital é uma dessas grandezas e atualmente é usada para apresentar a informação em um novo espaço.

Para a preservação de documentos, independentes de seus suportes, são geralmente levantados critérios e criadas ferramentas que proporcionem proteção e garantia para a sua manutenção, visando prevenir danos e minimizar riscos, assim como, restituir documentos que já tenham sido comprometidos (MÁRDERO ARELLANO, 2004).

As bibliotecas institucionais são peças centrais para o provimento da preservação digital. "Desde o início e meados da década de 1990, a coleta de informações entre as instituições gera novas práticas de preservação, restauração estratégias e colaboração institucional" (FINO-RADIN, 2011, p.6, tradução nossa). A preservação digital em seu real sentido usa visibilidades que se constituem em um novo paradigma para modernidade. Deste modo, focalizamos que a preservação digital e composta de:

[...] todas as atividades que são necessárias para manter o acesso a materiais digitais além dos limites da falha de mídia ou mudança tecnológica. Esses materiais podem ser registros digitais criados durante o dia-a-dia de negócios de uma organização, ou seja, materiais "born-digitais" criadas com um fim específico (por exemplo, recursos de ensino), ou os produtos de projetos de digitalização. (DIGITAL PRESERVATION COALITION, 2012, tradução nossa)

Com o advento das tecnologias da informação e comunicação, os organismos institucionais estão inteiramente ligados a natureza das tecnologias coorporativas e institucionais. Desta forma, a estrutura tecnológica de informação é comprometida diretamente na capacidade de processamento das informações pelo insumo de sua segurança e veracidade. "A Web é o maior documento já escrito, com mais de 4 bilhões de páginas públicas e uns 550.000.000.000 documentos conectados na Web" (LYMAN; VARIAN, 2000).

Deste modo, a sociedade da informação encontra-se em uma nova era de desafios e novas descobertas. Segundo Lyman (2002), 95\% da informação que está sendo criada nos anos recentes já se encontram em forma digital ou "nasceu digital". Para Márdero Arellano (2008, p. 36) "os novos suportes possibilitaram a transferência dos conteúdos informacionais para outras mídias totalmente diferentes do original”. Assim sendo, o estado da informação está definido pelo formato do suporte. Portanto, é simples a compreensão que os suportes que acoplam a informação, tenham em si a fragilidade, e a simplicidade de se corromperem. Para 
isto, as tecnologias se diversificam mudando seu valor no que se pode constatar o mesmo na preservação de materiais tradicionais.

A disponibilidade de produtos de informação digital baseadas na web está exercendo cada vez mais pressão sobre as bibliotecas tradicionais, que, por sua vez, estão cometendo porções maiores da sua dotação orçamental, quer para a aquisição ou a aceder a pesquisa on-line baseado na web ou de texto completo serviços, produtos CD-ROM, bases de dados on-line, produtos multimídia, etc. (ARORA, 2009, p.108, tradução nossa).

O principal objetivo da informação digital é melhorar o acesso à informação de alta qualidade. "A digitalização de documentos oferece benefícios duplos, preservar objetos raros e frágeis, assim como ele fornece maior acesso a vários números de usuários simultaneamente em locais remotos" (ARORA, 2009, p. 114, tradução nossa). As informações digitais são condicionadas a máquina. Pode não ser presumível acessar as informações a menos que tenha hardware adequado e software, para demandar acesso aberto.

A preservação digital refere-se a uma série de atividades, gerenciada e projetada para assegurar a continuidade de acesso a todos os tipos de registros em formatos digitais para o tempo necessário e para protegê-los de falha de mídia, a perda física e obsolescência (CORNELL UNIVERSITY LIBRARY, 2005). A sociedade da informação traz à tona as variáveis e facilidades de gerar informações e documentos. Isto é, com a perda dessas informações perder-se-ia a memória dos seres e da vida, o que deixaria uma grande lacuna na história, pois todo o conhecimento e a ciência podem deixar de permanecer por não haver o suporte e o material que as conserve ou as preserve, uma das preocupações são as que existem no mundo digital.

Tais informações, tornam-se grandes desafios ao conhecimento no ato de recuperá-las e contornam na preservação digital partículas de informações encapsuladas, da migração de suportes, para sua segurança e confiabilidade. Para Márdero Arellano (2004), na preservação digital o propósito da migração, é garantir a capacidade dos usuários de recuperar, exibir e utilizar os dados seguindo o desenvolvimento dos meios tecnológicos.

A gravidade disso, está exatamente no caso de transpor para novos formatos, preservando a integridade da informação modelo. Boeres e Arellano (2005, p. 10) apontam que "uma aceitável política de preservação digital implica em observar e aplicar procedimentos que podem ser inclusive aceitos como estratégias de preservação". Deste modo, a informação digital tem suas características e seus critérios na preservação digital, pautadas na recuperação da informação e nos seus suportes para provimento de acesso.

Assim sendo, Márdero Arellano (2008) discorre sobre os critérios da preservação digital e a define como "conjunto de atividades que determinam a manutenção de coleções digitais; elas definem as funções dos repositórios, os processos e procedimentos, a comunidade alvo, a usabilidade da informação e a infraestrutura técnica". Desta forma, Mazzarollo (2008, p. 3-7) aponta os principais critérios utilizados na preservação digital das quais alguns procedimentos podem ser constatados a seguir: 
1. Atualizar / Refrescamento: suporte físico que corre risco de se tornar obsoleto a ponto de nenhum outro periférico ser compatível com ele. Assim, ocasionando perda irreversível da informação. Para amenizar o problema, o refrescamento ou atualização de dados são requisitos para qualquer estratégia de preservação.

2. Emulação: processo que consiste em dispor de um sistema que funcione do mesmo modo que outro de tipologia diferente, para rodar programas. A emulação permite obter as características de determinado software ou hardware fielmente, sem nenhuma perda de dados.

3. Migração: consiste na transferência de dados na interface hardwaresoftware ou na interface atualização-obsolescência. Gerações de tecnologia digital sofrem constantes mudanças, e a migração também abrange esse tipo de salto tecnológico. Principalmente a atividade operacional mais utilizada por grandes instituições.

4. Encapsulamento: método de preservação digital que exige a observação do interesse do usuário para um estado no futuro, prevendo a necessidade de salvaguardar os dados exatamente como eles eram no momento de sua criação.

Assim, como foram ressaltadas as características de cada item do processo de preservação digital. Consequentemente, envolvem estratégias e adaptações para serem implantadas a respeito de cada técnica que é empregada na preservação digital. Por conseguinte, Ferreira (2006), aborda a vantagem de preservar as características e as funcionalidades do objeto digital original com um grau elevado de fidelidade. Desta forma, para o autor o objeto digital exige "camadas" de intermediação tecnológica, envolvendo a interface hardware-software, sem as quais a informação não pode ser acessada. A "camada" final, destinada aos usuários, depende das "camadas" anteriores, e estas são vulneráveis à obsolescência tecnológica.

Das estratégias de preservação, é importante ressaltar sua finalidade, a política da preservação digital está datada no último processo o de "encapsulamento" da qual seria separar as partes, mantê-las a mais isolada possível para que não haja perda de sua memória e suas informações. Segundo Mazzarollo (2008, p. 7), "a finalidade do encapsulamento é prevenir a futura perda de dados por simplesmente ignorar a informação no momento em que ela é criada".

\section{A PRESERVAÇÃO DIGITAL NO BRASIL}

A preservação digital é um assunto muito recente no momento e debatido na literatura da Ciências da Informação. Muitos autores fazem sua descrição, além de sua complexidade. Há muitos eventos, conferências nas regiões de todo Brasil sobre o assunto de política de preservação digital, repositórios institucionais, digitalização de documentos, sendo peça central das instituições públicas. Desta forma, ressalta-se que as comunidades produtoras e armazenadoras de documentos em arquivos digitais, devem estabelecer e praticar habitualmente uma política de preservação digital. (MÁRDERO ARELLANO, 2004, p. 24). 
Sendo assim, para Cunha (1999), nos últimos anos duas das funções básicas das bibliotecas estão sofrendo "perigo de extinção: a provisão de acesso à informação e a preservação do conhecimento para futuras gerações". Com o aumento da informação digital, tem sido alvo de questionamentos. Esta implicação está sobre o domínio dos órgãos e unidades em que se localizam essas informações, para a garantia e disponibilização em períodos de tempos (MÁRDERO ARELLANO, 2004, p.16).

No Brasil a preservação digital tem sido peça fundamental, nos repositórios das instituições de ensino das universidades e de institutos de pesquisa como IBICT, FBN e CONARQ. Essas instituições, tem se prontificado em promoverem programas e projetos voltados para a preservação do conhecimento. Tais projetos podem ser mencionados como SEER, a Rede Cariniana provida pelo IBICT e uma tentativa da Biblioteca Nacional e da CONARQ com objetivos, de conscientizar grupos que possuem arquivos de documentos digitais a adotar técnicas padronizadas de preservação digital.

No que concerne à preservação de documentos digitais, há uma preocupação nos formatos desses arquivos. Visando isto, a Organização das Nações Unidas para a Educação, a Ciência e a Cultura (UNESCO) publicou uma carta sobre a preservação de documentos digitais a partir disto a CONARQ constituiu critérios para assegurar o acesso posterior dos documentos digitais. Deste modo,

\begin{abstract}
As organizações públicas e privadas e os cidadãos vêm cada vez mais transformando ou produzindo documentos [...] exclusivamente em formato digital, como textos, bases de dados, planilhas, mensagens eletrônicas, imagens fixas ou em movimento, gravações sonoras, material gráfico, sítios da internet, dentre muitos outros formatos e apresentações possíveis de um vasto repertório de diversidade crescente. (BRASIL, 2006, p. 2).
\end{abstract}

As políticas de preservação digital nas instituições públicas têm em seu percurso, a aplicabilidade de procedimentos da qual sugere as estratégias de preservação de documentos. Os institutos brasileiros já se organizam para dar início a operabilidade de digitalização. O Instituto de Brasileiro de Informação Ciência e Tecnologia (IBICT), na tentativa de salvaguarda registros da informação científica e do patrimônio cultural no Brasil, buscam várias parcerias com diversas instituições á fora em busca de softwares que melhor preserve digitalmente a informação em longo prazo.

Desses sistemas, contextualizamos alguns como a LOCKSS, DAITSS, Fedora, Dspace, ICA-ATOM, Archivematica. Podemos ressaltar atualmente a LOCKSS, Lot of Copies Keep Stuffs Safe (Muitas Cópias Mantêm Coisas Seguras). É o software criado pela equipe LOCKSS da Universidade de Stanford, originalmente desenvolvido para a preservação de periódicos eletrônicos (MÁRDERO ARELLANO, 2008, p.115).

O Open Journal Systems (OJS), software livre, de fonte aberta, desenvolvido pelo Public Knowledge Project originário da British Columbia University (Canadá) foi personalizado e traduzido pelo Instituto Brasileiro de Informação em Ciência e 
Tecnologia (Ibict), com o nome de SEER (Sistema Eletrônico de Editoração de Revistas) (MÁRDERO ARELLANO, 2008, p.119).

Inteiramos, que deste 2002 o IBICT vem tendo a iniciativa com a parceria do programa LOCKSS da Stanford University e 9 instituições de Ensino Superior entre universidades Federais e Estaduais, com objetivo de salvaguardar os documentos eletrônicos brasileiros. A Rede Cariniana provida pelo IBICT é uma organização colaborativa e "tem por objetivo preservar as revistas eletrônicas que utilizam a plataforma OJS/SEER no Brasil”. (INSTITUTO BRASILEIRO DE INFORMAÇÃO CIÊNCIA E TECNOLOGIA, 2013).

Deste modo, a Rede Brasileira de Preservação Digital é um organismo cooperativo de vários colaboradores. A rede é um processo de coleta distribuída de publicações eletrônicas, e tem por objetivo preservar as publicações e seus conteúdos a longo prazo para as futuras gerações. Complementa-se que a Rede Cariniana deu início a preservação dos periódicos eletrônicos das instituições de Ensino Superior parceiras do projeto que utilizam a plataforma OJS/SEER.

Ressalta-se que os próximos passos da Rede Cariniana será a ampliação de seus serviços de preservação, como Teses e Dissertações das instituições que utilizam o software Dspace. E deste modo, foca-se em potencializar as atividades humanas que já estão sendo orientadas para facilitar a automatização dos processos de digitalização, métodos de estocagem, autenticidade e conversão para novos formatos digitais.

\section{BIODIVERSITY HERITAGE LIBRARY}

Biodiversity Heritage Library (BHL) é uma Associação Internacional formada por museus de história natural, jardins botânicos, biblioteca e centros de pesquisa biológica. Cujo objetivo é contribuir para digitalização e publicação de acesso aberto de obras ricas em biodiversidade realizada em suas respectivas coleções sendo o acesso e a publicação como partes de um "Universo Comum em Biodiversidade".

Segundo Kasperek (2010, p. 448, tradução nossa) a BHL foi "fundada em 2005, e tornou-se o terceiro maior projeto de digitalização para literatura sobre biodiversidade [...] atualmente a BHL é o maior projeto de digitalização na sua temática”.

\footnotetext{
A BHL trabalha com a comunidade internacional taxonômica, os detentores de direitos e outras partes interessadas para garantir que este patrimônio de biodiversidade é disponibilizado para uma audiência global através de princípios de acesso aberto. A BHL obteve permissão de editores para digitalizar e tornar disponíveis materiais de biodiversidade significativos que ainda estão sob os direitos autorais [...]. Em parceria com a Internet Archive e através de esforços de digitalização locais, a BHL digitalizou milhões de páginas de literatura taxonômica, o que representa dezenas de milhares de títulos e mais de 100.000 volumes. Para adquirir conteúdo adicional e promover o livre acesso à informação. (BIODIVERSITY HERITAGE LIBRARY, 2014, online, tradução nossa).
} 
O conhecimento sobre a diversidade natural não tem se limitado apenas a um grupo seleto de bibliotecas. As coleções de obras de biodiversidade são de valor inusitado, posto que seja dos domínios biológicos sobre as suas sistematizações que envolvem a Ciência e o conhecimento acerca da literatura e sua história.

Do mesmo modo, Santos e Silva (2006, p. 6) raciocinam que:

Na sociedade contemporânea, a informação desempenha um importante papel nas relações entre os seres humanos, inserida que está nas atividades intelectuais, financeiras, comerciais e pessoais, disponibilizada por meio de uma diversidade de formatos e suportes. A sua produção, organização, domínio e transformação podem causar mudanças políticas, culturais e econômicas de uma região ou nação.

A informação bibliográfica ambiental é rica, porém poucos têm acesso a essa gama de conhecimento. Todavia, esta abundância de informação está acessível apenas para aqueles poucos que podem ter passagem direta ao acervo das bibliotecas. Para a Biodiversity Heritage Library, (2014, online, tradução nossa) o acesso livre ao conhecimento em biodiversidade, permutará coleções entre as das mais diversas. Suas metas consistem das seguintes maneiras:

1. Conteúdo relevante: construir e manter a BHL como o maior repositório confiável, respeitável, e responsivo de literatura biodiversidade e materiais de arquivo.

2. Ferramentas e Serviços: desenvolver serviços e ferramentas que facilitam a descoberta e melhorar a eficiência do conteúdo BHL pesquisa.

3. O envolvimento dos usuários: aumentar a conscientização global sobre a BHL através de divulgação, aprendizagem e educação, e marca por meio do engajamento e colaboração com as comunidades de usuários novos e existentes.

4. Participação e Parcerias: crescer BHL adesão e parcerias consórcios, promover a colaboração entre instituições que continua a servir de modelo para o desenvolvimento da biblioteca digital.

5. Sustentabilidade Financeira: garantir a sustentabilidade e relevância por ser flexível, adaptável e financeiramente sólida, enquanto o conteúdo e os serviços permanecem aberta e livremente disponíveis.

A rede BHL tem sua preocupação com a preservação dos acervos em biodiversidade e meio ambiente. Desta forma, a maior parte de seu acervo coletivo, já se encontra publicado em seu Portal para acesso livre para profissionais e pesquisadores. O portal BHL é um grande empreendimento, para preservação de uma herança histórica na natureza e da vida. Deste modo, "o Portal BHL permite aos usuários pesquisar a coleção em biodiversidade por vários pontos de acesso, podendo ler os textos on-line, ou baixar páginas selecionadas ou volumes inteiros como arquivos com formato TIF, JP2, OCR, ALL e PDF" (BIODIVERSITY HERITAGE LIBRARY, 2014, online, tradução nossa).

A BHL tem como intuito colaborar para a competência, inclusão, divulgação, obtenção e interoperabilidade da informação científica em Biodiversidade no mundo. Neste sentido, disponibiliza documentos com informações sobre mais de 150 milhões de designações de espécies entre animais e plantas, usando a Global Names Recognition and 
Discovery (GNRD) e os nomes de taxionomias para encontrá-las. Deste modo, os pesquisadores podem reunir publicações sobre as espécies e links para conteúdo listado, trabalhando desta forma, com uma verdadeira enciclopédia em ciências naturais.

\begin{abstract}
Desde 2009, a BHL se expandiu globalmente. EContentPlus programa da Comissão Europeia financiou o projeto BHL- Europa, com 28 instituições, para montar a literatura de língua Europeia. Além disso, a Academia Chinesa de Ciências (BHLChina), a Atlas of Living Australia (BHL- Austrália), Brasil (através BHLSciELO) e da Biblioteca Alexandrina criaram laços nacionais ou regionais com a BHL. Além disso, em abril de 2013, o nó mundial BHL- África foi lançada oficialmente. Nós globais são estruturas organizacionais que podem ou não podem desenvolver seus próprios portais BHL. O objetivo da BHL é compartilhar e servir conteúdo por meio do Portal BHL desenvolvido e mantido no Missouri Botanical Garden. Todas essas parcerias vão trabalhar juntos para compartilhar conteúdo, protocolos, serviços e práticas de preservação digital (BIODIVERSITY HERITAGE LIBRARY, 2014, online, tradução nossa).
\end{abstract}

A BHL busca a conservação digital de um grande domínio natural, e a expansão da informação científica. Uma vez que é imprescindível sua importância, pois além da informação em biodiversidade, faz com que preserve a informação e a torne possível, disponível a todos. Sendo do impresso ao formato digital, em operações de acesso aberto e das redes corporativas e do enorme patrimônio que chamamos de biodiversidade.

O projeto BHL segue em rumo aos vários continentes, a América Latina é umas das escolhidas para projeto em parceria com SCIELO, Projeto BHL Brasil. Com 7 instituições participantes. Deste modo, o projeto prevê a interoperabilidade internacional de sistemas, produtos e serviços de informação e comunicação científica em biodiversidade, particularmente na Biodiversity Heritage Library, onde estão publicados os acervos já digitalizados e em acesso aberto e os demais acervos que serão digitalizados e alocados no portal para acesso.

\title{
5 PRESERVAÇÃO DIGITAL EM COLEÇÕES BIBLIOGRAFICAS: O CASO DO MUSEU PARAENSE EMÍLIO GOELDI
}

A biblioteca do Museu Paraense Emilio Goeldi, recebe o nome de Domingos Soares Ferreira Penna, em homenagem a um dos antigos diretores da instituição. É especializada nas áreas de Botânica, Ciências Naturais e da Terra, Antropologia e áreas de intervenção do Museu relacionadas com a Amazônia. Têm por objetivo reunir, selecionar, processar, armazenar, preservar e divulgar o material bibliográfico e conhecimento nas áreas especializadas do Museu Goeldi sobre a Amazônia.

Tem como escopo institucional, contribuir para o estudo, a pesquisa e a geração do conhecimento e o desenvolvimento cultural tanto dos seus funcionários como de seus pesquisadores, alunos de pós-graduação e professores pertencentes, também, à outras instituições de pesquisa em território nacional e internacional. Desempenha atividades com o propósito de lidar com material bibliográfico, quando adquiridos pela instituição até que seja disponível para o usuário final, que pode ser um pesquisador, estudante de pós-graduação, 
bolsistas de pesquisa, etc. O conhecimento produzido pelo Museu Goeldi é transformado em livros científicos e revistas, que carregam a informação para o mundo do conhecimento.

Deste modo, na atual composição de atividades da biblioteca ressaltamos a atividade do projeto de digitalização de acervos BHL/SCIELO. Sob essa ótica, o projeto tem como intuito colaborar para a competência, relação inclusão, divulgação, obtenção e interoperabilidade da informação científica em Biodiversidade. De forma resumida, seu propósito é disponibilizar documentos com informações sobre mais de 150 milhões de designações de espécies. Usando a Global Names Recognition and Discovery (GNRD) e os nomes de taxionomias para encontrá-las.

Desta forma, relatamos o início das atividades regidas das etapas da digitalização do das Obras Raras em Biodiversidade. Assim sendo, a digitalização das obras na Biblioteca do Museu Emilio Goeldi tem a finalidade de descrever as ações práticas do processo da digitalização no Museu Goeldi. Assim, o projeto de digitalização mostra a sua praticidade no processo de viabilidade no acervo em Biodiversidade no Museu Emílio Goeldi. Nesta perspectiva, o projeto segue com a parceria da SCIELO com 7 instituições participantes. Para alocar 2.000 obras do século XVIII e XIX no portal BHL Brasil visando prover o acesso a informação cientifica e a preservação das publicações digitais em biodiversidade.

Portanto, contextualizamos que o foco do projeto de digitalização no Museu Goeldi e os processos das obras bibliográficas. Tem almejado, até o presente momento a análise da digitalização dos periódicos antigos do Museu Goeldi, bem como os Boletins do Museu Paraense Emílio Goeldi datado de 1880 até 1911 e recolhidos para a coleção especial.

\begin{abstract}
O Boletim do Museu Paraense Emílio Goeldi é a principal publicação da instituição. Concebido por Emílio Goeldi, em 1894, seu primeiro volume foi lançado em 1896 com o nome de Boletim do Museu Paraense de História Natural e Ethnografia. O volume 4, fascículo 1 (1904), foi publicado como Boletim do Museu Goeldi de História Natural e Ethnografia e, desde o volume 10 (1949), como Boletim do Museu Paraense Emílio Goeldi, em homenagem a seu idealizador. A nova série foi iniciada em 1957 e continuada até 1984, quando uma nova numeração dos volumes foi adotada. O atual Boletim do Museu Paraense Emílio Goeldi é editado em quatro séries: Antropologia, Botânica, Zoologia (semestral) e Ciências da Terra (anual) (VARELA; BAIÃO NETO, 2012, p.6).
\end{abstract}

Partindo dessa perspectiva a digitalização dos boletins foi analisada a partir de uma amostra do processo de digitalização limitando a pesquisa. Foram feitos treinamentos para realizar as atividades de digitalização que foram promovidos pela Scielo.

A Tabela 1 mostra a fase inicial da digitalização no Museu Goeldi, e os Boletins que já foram digitalizados, assim como a quantidades de páginas.

TABELA 1- Boletins Digitalizados

\begin{tabular}{llll}
\hline Boletim do Museu Goeldi $\quad$ Volume & Número & Ano & $\begin{array}{l}\mathbf{N}^{\circ} \text { de Páginas } \\
\text { aproximado }\end{array}$
\end{tabular}

$\begin{array}{lllll}\text { História Natural e Ethinographia } & 1-10 & 1-4 & 1896-1949 & 3.782\end{array}$



Zoologia
$1-20$
$1-124$
$1964-2002$
2.798
Botânica
$1-20$
$1-59$
$1954-2002$
4.864

Total de páginas digitalizada

11.444

Fonte: Dados da Pesquisa, 2014.

Como perspectiva, a contribuição desse estudo advém de 12 meses de muito trabalho na biblioteca do Museu Emílio Goeldi, onde as publicações periódicas foram digitalizadas. $\mathrm{O}$ próximo passo para os próximos 12 meses, se resume a digitalização da maior parte da coleção, referente aos livros raros e infólios.

Das técnicas e políticas de digitalização que foram estabelecidas destacamos a) o processamento de imagens digitalizadas, b) descrição bibliográfica e indexação das obras, c) manuais para tratamento das obras digitalizadas, c) tratamento de texto reconhecido, armazenamento, com o objetivo apresentar, restaurar e preservar no meio digital.

Das políticas de operação realizada no processo da digitalização foi estabelecido a 1) definição de método para o tratamento de obras raras e o controle da literatura, 2) armazenamento estruturado e recuperação de documentos indexados 3) políticas e procedimentos para a gestão da propriedade intelectual da coleção como um todo e os domínios de acesso 4) utilização de acordo com a metodologia de Creative Commons. 5) composição de um conjunto de web set com protocolos abertos para permitirem acesso online e uso de outros produtos e serviços da rede web.

A partir da tomada dessas políticas, as obras digitalizadas estão inteiramente armazenadas e preservadas para duas versões, com baixa resolução que marca a apresentação em telas de computador e de alta resolução para preservação e uso em alta resolução em dispositivos de impressão.

O acervo bibliográfico especial é atualmente dividido em uma coleção sobre a biodiversidade, entre livros, infólios, revistas. Em meio a estes destacamos alguns que estão sendo digitalizados: Os Boletins antigos do Museu Paraense Emílio Goeldi, de História Natural e Ethinographia, Zoologia, Botânica, publicações avulsas do Museu Goeldi, Goeldiana, On infólios Un voyage botanique dans les bas-Amazone de Raymond Bouillenne de 1930, Mamíferos da Amazônia de 1944 e Mammals of Amazônia de 1945. Apoio do Museu Paraense Emilio Goeldi de História Natural e Etnografia contribuição de Eládio da Cruz Lima 1900-1943 estas e outras publicações estão sendo digitalizadas.

Atualmente o processo de digitalização em acervos bibliográficos em bibliotecas tem produzido ações para melhor acesso ao público, tendo em vista, o objetivo de salvaguardar as informações contidas em seu formato físico para o digital, já que o formato em papel por exemplo, quando não cuidado e preservado terá tendência natural a se degenerar. 
Por isso, contextualizamos a importância de preservar digitalmente essa coleção. Com a atuação do projeto BHL no Museu Emílio Goeldi. Observa-se que as tecnologias se tornaram ferramentas poderosas para a preservação da memória e a transmissão de informações.

\section{CONSIDERAÇÕES FINAIS}

A preservação no universo digital é uma tarefa desafiadora para bibliotecários e arquivista e os profissionais da informação. No entanto, os protocolos, estratégias e tecnologias envolvidas na preservação digital já foram bem definidos e compreendidos. A preservação digital é uma atividade de alto custo para as bibliotecas, arquivos ou museus. Para sua natureza podem tomar a decisão de adotar a digitalização com preservação em longo prazo e armazenamento de coleções de pesquisa, sem profundo e contínuo compromisso com a preservação pela instituição de origem.

A informação digital forma uma parte cada vez maior do nosso patrimônio cultural e intelectual e oferece benefícios significativos para os usuários. Ao mesmo tempo a preservação e o acesso livre a informação é dependente de meios e tecnologias das quais são impermanente tendo em vista a retenção de metadados sobre a proveniência de seu contexto, mantendo a autenticidade e o conteúdo dos mesmos.

E nesta perspectiva o Museu Paraense Emílio Goeldi como instituição participante do projeto BHL teve em seus critérios, políticas e procedimentos definidos para a formação e o desenvolvimento da coleção de obras raras em biodiversidade. Embora a experiência da digitalização e dos dados digitais tenham sido construídos, nos resultados obtidos é relativamente novo seu meio, onde a maior parte do ciclo de vida, atividades e custos futuros e modelos são atualmente um caminho em construção.

Neste sentido, esses fatores levam a crescente preocupação com a potencial perda de nossa "memória coletiva" na Era Digital, para instigar mais a investigação sobre a preservação em longo prazo para a informação digital se manter para as futuras gerações. Desta forma, os recursos digitais, sem dúvida, têm várias vantagens sobre seu contador analógico em partes, no entanto, a preservação não é definitivamente um deles.

A ocorrência de que o risco de perda de dados em formato digital é muito maior do que qualquer outra forma física é bem compreendida e dirigida. A preservação em longo prazo da informação digital é balizada pela curta vida de mídia, hardware e software obsoleto e de leitura lenta de velha mídia. No entanto, além das dificuldades de mídia, perda de informação e de sua curta vida média.

Contudo, apresentamos que o processo de digitalização está em andamento, tendo em vista resultados preliminares de 12 meses de experiência. Desta forma, acreditamos que este trabalho tem feito contribuições significativas como a disponibilidade e o conhecimento do objetivo proposto, desde que seu objetivo seja de continuar a preservação da memória digital na Amazônia. 
Sobretudo, ressaltamos que haverá os resultados finais que sobrevirão para que seja constatado e autenticado como experiência e visibilidade nos centros de memória. Mostrando as atividades e as etapas de digitalização das coleções e as ações de práticas de preservação digital do projeto de digitalização na Biblioteca do Museu Emílio Goeldi.

\section{REFERÊNCIAS}

ARORA, Jagdish. Digital preservation: an overview. INFLIBNET, Nova Deli, 29-30 jan. 2009. p.116-146. Disponível em: <http://ir.inflibnet.ac.in/bitstream/1944/1466/1/8.pdf>. Acesso em: 19 jan. 2014.

BABBIE, Earl. The practice of social research. California: Wadsworth Publishing Company, 1983.

BIODIVERSITY HERITERAGE LIBRARY. Wiki: tips and information for our users, 2014. Disponível em: <http://biodivlib.wikispaces.com>. Acesso em: 12 de janeiro de 2014.

BOERES, Sonia A. de Assis; ARELlANO, Miguel A. Márdero. Políticas e estratégias de preservação de documentos digitais. In: CinForm, 4. Proceedings... Salvador, 2005. Disponível em: $<$ http://www.cinform.ufba.br/vi_anais/docs/SoniaMiguelPreservacaoDigital.pdf $>$. Acesso em: 15 nov. 2013.

BRASIL. Conselho Nacional de Arquivos. Modelo de requisitos para sistemas informatizados de gestão arquivística de documentos: e-ARQ. Rio de Janeiro: CONARQ, 2006. Disponível em: $<$ http://www.arquivonacional.gov.br/conarq/cam_tec_doc_ele/gestao/e-ARQ\%20\%20v\%200.pdf>. Acesso em: 10 jan. 2014.

CORNELL UNIVERSITY LIBRARY. Tutorial on digital preservation management: implementing short-term strategies for long-term problems, 2005. Disponível em: < http://www.library.cornell.edu/iris/dpworkshop/instructors.html >. Acesso em: 19 jan. 2014.

CUNHA, Murilo Bastos da. Desafios na construção de uma biblioteca digital. Ciência da Informação, Brasília, v. 28, n. 3, p. 257-268, set./dez. 1999. Disponível em: $<$ http://www.ibict.br/cienciadainformacao/rst/viewarticle.php?id=323 > . Acesso em: 28 jan. 2014

DIGITAL PRESERVATION COALITION. Digital preservation coalition. Disponível em: $<$ http://www.dpconline.org/ > . Acesso em: 04 jan. 2014.

FERREIRA, Miguel. Introdução à preservação digital: conceitos, estratégias e actuais consensos. Portugal: Escola de Engenharia da Universidade de Minho, 2006. Disponível em: 
$<$ https://repositorium.sdum.uminho.pt/bitstream/1822/5820/1/livro.pdf $>$ Acesso em: 12 jan. 2014.

FINO-RADIN, Ben. Digital preservation practices and the rhizome artbase. Rhizome.org, 2011. Disponível em: <http://rhizome.org/editorial/2011/aug/5/keeping-it-online/>. Acesso em: 04 jan. 2014.

GIL, Antonio Carlos. Como elaborar projetos de pesquisa. 4. ed. São Paulo: Atlas, 2008.

INSTITUTO BRASILEIRO DE INFORMAÇÃO CIÊNCIA E TECNOLOGIA. Guia de preservação digital de periódicos eletrônicos na rede cariniana. Brasília: IBICT, nov. 2013. Disponível em: <https://attachment.fbsbx.com/file_download.php?id=4700829964376 42\&eid=ASvdD2nISTf1tEgMpTqeBaJVo36wmPpdG_xWvEOiDvvoaChmUd_a7Xw63zlj4e

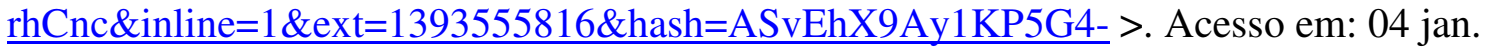
2014.

KASPEREK, Gerwin. Eine Übersicht von für die Biologie relevanten Projekten zur Digitalisierung historischer Fachliteratur: Darstellung eines speziellen Segmentes aus dem Internetquellen-Führer einer Virtuellen Fachbibliothek. Bibliotheksdienst, v.44, n. 5, p. 448460, maio. 2010. Disponível em: < http://www.degruyter.com/view/j/bd.2010.44.issue5/bd.2010.44.5.448/bd.2010.44.5.448.xml >. Acesso em: 15 jan. 2014.

LYMAN, P. Archiving the world wide web: in building a national strategy for preservation: issues in digital media archiving. Washington: Council on Library and Information Resources, 2002. Disponível em: <www.clir.org/pubs/reports/pub106/web.html>. Acesso em: 14 jan. 2014.

LYMAN, Peter; VARIAN, Hal R. How much information? Regents of the University of California, 2000. Disponível em: <http://www.sims.berkeley.edu/research/projects/howmuch-info/.>. Acesso em: 14 jan. 2014.

MANZO, Abelardo J. Manual para La preparación de monografias: uma guia para presentar informes y tesis. Buenos Aires: Humanitas, 1971.

MÁRDERO ARELLANO, Miguel Ángel. Critérios para a preservação digital da informação científica. 2008. 354 f. Tese (Doutorado em Ciência da Informação) Universidade de Brasília, Brasília, 2008. Disponível em: $<$ http://hdl.handle.net/10760/12649>. Acesso em: 15 fev. 2014.

Preservação de documentos digitais. Ciência da Informação, v.33, n. 2, p. 15-27, maio/ago. 2004. Disponível em: <http://revista.ibict.br/index.php/ciinf/article/viewArticle/305/270> . Acesso em: 12 jan. 2014.

MAZZAROLLO, Fabiana. Estratégias de preservação digital. Porto Alegre: UFRGS, 2008. Disponível em: < http://fabianamazzarollo.files.wordpress.com/2008/11/estrategias-depreservacao-digital.pdf $>$. Acesso em: 12 jan. 2014. 
PIOVESAN, A. Da necessidade das escolas de saúde pública elaborarem métodos simplificados de investigação social. S. Paulo, 1968. Tese (Doutorado) - Faculdade de Saúde Pública da USP.

QUEIRÓZ, M. I. de P. O pesquisador, o problema da pesquisa, a escolha de técnicas: algumas reflexões. In: Lang, A.B.S.G.( Org.) Reflexões sobre a pesquisa sociológica. São Paulo, Centro de Estudos Rurais e Urbanos, 1992. p. 13-29. (Coleção Textos; 2a série, $3)$.

SANTOS, Fernando Bittencourt dos; SILVA, Helen de Castro. Busca e uso da informação ambiental por pesquisadores da área de meio ambiente. In: FÓRUM AMBIENTAL DA ALTA PAULISTA, 2., 2006, São Paulo. Disponível em:<

http://www.amigosdanatureza.org.br>. Acesso em: 10 jan. 2014.

SANTOS, Vanderlei Batista dos; INNARELLI, Humberto Celeste; SOUSA, Renato Tarcísio Barbosa de. Arquivística: temas contemporâneos. 3. ed. Brasília, D.F.: SENAC, 2009.

SARACEVIC, Tefko. Ciência da informação: origem, evolução e relações. Perspectivas em ciência da informação, Belo Horizonte, v. 1, n. 1, p. 41-62, jan./jun. 1996. Disponível em: $<$ http://portaldeperiodicos.eci.ufmg.br/index.php/pci/article/viewFile/235/22>. Acesso em: 15 jan. 2014.

TRUJILLO FERRARI, Alfonso. Metodologia da ciência. 3. ed. Rio de Janeiro: Kennedy, 1974.

VARELA, Alex; BAIÄO NETO, Gil. Museu Paraense de História Natural e Etnografia. Dicionário Histórico-Biográfico das Ciências da Saúde no Brasil, 2012. Disponível em: < http://www.dichistoriasaude.coc.fiocruz.br/iah/pt/pdf/muspareg.pdf $>$. Acesso em: 12 jan. 2014.

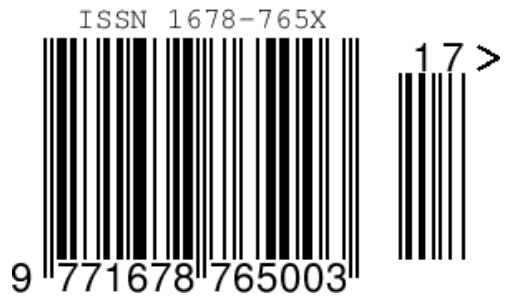

CASTRO, Jetur Lima de; OLIVEIRA, Alessandra Nunes de. Preservação digital em coleções bibliográficas da biodiversidade: o caso da Biodiversity Heritage Library no Museu Paraense Emílio Goeldi (MPEG). RDBCl: Revista Digital de Biblioteconomia e Ciência da Informação, Campinas, SP, v. 14, n. 1, p. 192-207, dez. 2015. ISSN 1678-765X. Disponível em: <http://periodicos.sbu.unicamp.br/ojs/index.php/rdbci/article/view/8642124/9926>. Acesso em: 01 fev. 2016. doi:http://dx.doi.org/10.20396/rdbci.v14i1.8642124. 